\title{
Global and Regional Myocardial Innervation Before and After Ablation of Drug-Refractory Ventricular Tachycardia Assessed with ${ }^{123}$ I-MIBG
}

\author{
Mohammed Abdulghani ${ }^{1,2}$, John Duell ${ }^{1,2}$, Mark Smith ${ }^{1,3}$, Wengen Chen ${ }^{1,3}$, Søren M. Bentzen ${ }^{4}$, Ramazan Asoglu ${ }^{1,2}$, \\ Tomas Klein ${ }^{1,2}$, Tamunoinemi Bob-Manuel ${ }^{1,2}$, Anastasios Saliaris ${ }^{1,2}$, Vincent See ${ }^{1,2}$, Stephen Shorofsky ${ }^{1,2}$, \\ Vasken Dilsizian $^{1,3}$, and Timm Dickfeld ${ }^{1,2}$ \\ ${ }^{I}$ Maryland Arrhythmia and Cardiology Imaging Group, Division of Cardiology, University of Maryland School of Medicine, \\ Baltimore, Maryland; ${ }^{2}$ Department of Medicine, University of Maryland School of Medicine, Baltimore, Maryland; ${ }^{3}$ Department \\ of Diagnostic Radiology and Nuclear Medicine, University of Maryland School of Medicine, Baltimore, Maryland; and ${ }^{4}$ Department \\ of Epidemiology and Public Health, University of Maryland School of Medicine, Baltimore, Maryland
}

Cardiac innervation is a critical component of ventricular arrhythmogenesis that can be noninvasively assessed with ${ }^{123}$-MIBG. However, the effect of ventricular tachycardia (VT) ablation on global and regional left ventricular sympathetic innervation and clinical outcomes has not been previously assessed. Methods: In this prospective, single-center feasibility study, 13 patients with cardiomyopathy $(n=$ 9 ischemic, $n=4$ nonischemic) who were scheduled to undergo ablation of drug-refractory VT underwent 15-min and 4-h ${ }^{123}$ I-MIBG scans before and $6 \mathrm{mo}$ after the ablation procedure. Planar and arrhythmia-specific 757-segment analysis of short-axis SPECT images was performed in all datasets. Results: Global innervation assessed with heart-to-mediastinal ratio and washout rates was preserved in all patients at baseline (1.8 [continuous variables are expressed as median and quartile: Q1-Q3, 1.7-2.4] and 54\% [Q1-Q3, 47\%-67\%]) and did not change significantly at the 6-mo follow-up (1.9 [Q1-Q3, 1.62.2], $P=0.9$; and 56\% [Q1-Q3, 41\%-62\%], $P=0.6$ ). However, segmental analysis demonstrated that ischemic patients had larger areas of abnormal innervation at baseline $(52.1 \%$ vs. $19.6 \%, P=$ $0.011)$ and at the 6 -mo follow-up $(56.7 \%$ vs. $27.5 \%, P=0.011)$ than the nonischemic patients. Innervation defects affected $40 \%$ of the inferior segments in all ischemic cardiomyopathy patients, whereas they affected only $10 \%$ of inferior segments in $75 \%$ of nonischemic patients. When segmental data were further analyzed in denervated (DZ), transition (TZ), and normal (NZ) zones, there were changes in these designated innervation categories from baseline to the 6-mo follow-up for ischemic (19\% DZ, 59\% TZ, 22\% NZ) and nonischemic ( $6 \% \mathrm{DZ}, 45 \% \mathrm{TZ}, 15 \% \mathrm{NZ})$ patients. In ischemic patients, relative changes were significantly greater in the TZ segments than in the $D Z$, which demonstrated the second highest proportional changes $(P=0.028)$. Receiver operating characteristic curves defined best cutoffs of DZ, TZ, and NZ as less than $30.5 \%, 30.6 \%-47.1 \%$, and more than $47.1 \%$, respectively. Conclusion: Patients with ischemic cardiomyopathy have larger areas of abnormal innervation than those with nonischemic cardiomyopathy. Although VT ablation did not change global innervation, a novel arrhythmia-specific segmental analysis demonstrated significant dynamic changes in innervation categories and allowed quantitative definitions of DZ, TZ, and NZ.

Received Feb. 12, 2015; revision accepted Apr. 17, 2015.

For correspondence or reprints contact: Timm Dickfeld, Division of Cardiology, University of Maryland School of Medicine, 22 S. Greene St., Baltimore, MD 21201.

E-mail: tdickfel@medicine.umaryland.edu

COPYRIGHT (c) 2015 by the Society of Nuclear Medicine and Molecular Imaging, Inc.
These findings provide novel insights into the mechanics of sympathetic innervation in patients undergoing VT ablation and may have diagnostic and therapeutic implications.

Key Words: ${ }^{123}$ I-MIBG; ventricular tachycardia ablation; cardiac innervation; radionuclide imaging; cardiomyopathy

J Nucl Med 2015; 56:52S-58S

DOI: 10.2967/jnumed.115.155143

$\mathbf{R}$ eentrant ventricular tachycardia (VT) in structural heart disease is mediated through the functional VT substrate consisting of fixed, extracellular fibrosis as well as intermixed, electrically remodeled myocardium (1). Current ablation techniques mostly target the anatomic substrate in the form of scar tissue with surviving channels of myocardium that act as necessary pathways of slow conduction (2). However, the success of this approach remains limited, with a VT recurrence rate of $47 \%$ over 6 mo in one series despite stateof-the-art technology used by experienced operators (3).

One possible explanation is that current imaging techniques do not take into consideration the triggering mechanisms and substrate modulators, such as sympathetic cardiac innervation (4-9). The relationships between cardiac autonomic nervous system dysfunction, cardiomyopathy, and cardiac arrhythmias have been long established (10-12). Cardiac sympathetic innervation can be directly imaged with ${ }^{123}$ I-meta-iodobenzylguanidine ( $\left.{ }^{123} \mathrm{I}-\mathrm{MIBG}\right)$, a radiolabeled norepinephrine analog $(13,14)$. Multiple studies using global parameters to evaluate cardiac sympathetic innervation have demonstrated that innervation is associated with implantable cardioverter-defibrillator (ICD) therapies, ventricular arrhythmias, and sudden cardiac death in patients with both ischemic cardiomyopathy (ICM) and nonischemic cardiomyopathy (NICM) (15-17). However, little is known regarding the impact of VT ablation on dynamic changes of innervation, or whether these processes differ among different cardiomyopathies.

Because innervation is a regional process, greater resolution may be needed than is afforded with global measurements or the traditional 17-segment model, which was designed to detect coronary artery disease in the 3 major vascular territories (18). The components of the reentrant circuits that sustain scar-mediated VT can be much smaller 
and may not be appreciated on a standard 17-segment polar plot. Furthermore, loss of sympathetic innervation will frequently extend beyond the zone of myocardial infarction in patients with ICM (19) and may not follow coronary distribution patterns at all in patients with NICM. Consequently, innervation will likely be heterogeneous even within a given segment in the 17 -segment model. These drawbacks of the 17-segment model may in part explain why SPECT results did not demonstrate any prognostic benefit in the ADMIRE$\mathrm{HF}$ trial (20). Additionally, segment sizes of the 17-segment model do not have the resolution to analyze the effects of ablation with catheter tips as small as $3.5 \mathrm{~mm}$. Hence, this study sought to demonstrate the feasibility of using a higher-resolution, arrhythmia-specific, 757segment ${ }^{123}$ I-MIBG polar plot to evaluate cardiac sympathetic innervation in patients with recurrent VT and the effects of VT ablation on regional innervation.

\section{MATERIALS AND METHODS}

All study protocols were reviewed and approved by the University of Maryland Institutional Review Board, and all patients provided written, informed consent. The study was designed as a prospective, single-center feasibility study of patients with cardiomyopathy (ischemic and nonischemic) who underwent ablation for drug-refractory scar-mediated VT at the University of Maryland Medical Center (Baltimore, MD) from August 2010 through April 2013. Patients with normal heart VT were excluded. Patients were defined as having ICM if they had a history of a myocardial infarction or a significant $(>70 \%)$ stenosis in one or more major coronary arteries.

\section{VT Ablation}

The ablation procedures targeted clinical VT as documented by $12-$ lead electrocardiograms when available, or presumed clinical VT defined by cycle lengths, local right ventricular timing to far-field electrograms, and far-field morphology from ICD recordings. Activation mapping was performed if the VT was sustained and hemodynamically tolerated. For hemodynamically unstable or nonsustained VT, pace map matches of $11 / 12$ or more, with the longest stimulusQRS (if multiple sites with identical match are found) defining the site closest to the central isthmus, were used to approximate the VT channel and exit site. Radiofrequency ablation lesions (40-50 W, $60 \mathrm{~s}$ ) were applied at these locations. Additional VT substrate modification was performed if activation mapping and pace mapping did not demonstrate a suitable ablation site, or as otherwise clinically indicated. At the end of the ablation, programmed electrical stimulation was repeated, and successful ablation was defined as the inability to induce the clinical or presumed clinical VT.

\section{I-MIBG Image Acquisition and Analysis}

${ }^{123}$ I-MIBG planar and SPECT image acquisition was performed in 13 patients with drug-refractory VT before and 6 mo after VT ablation. Patients were pretreated with either perchlorate (potassium or sodium) or an iodine solution at least $60 \mathrm{~min}$ before injection to block uptake of free iodine by the thyroid gland. Patients were administered approximately $370 \mathrm{MBq}(10 \mathrm{mCi})$ of ${ }^{123} \mathrm{I}-\mathrm{MIBG}$ (GE Healthcare) intravenously. Planar imaging of the anterior thorax $(128 \times 128$ matrix $)$ was performed 15 min later, as was SPECT imaging using a dual-head $\gamma$ camera (minimum of 30 projections per head, 20 to $30 \mathrm{~s}$ per projection, $64 \times 64$ matrix, SKYLight SPECT camera [Philips]). Repeat planar and SPECT imaging was performed $4 \mathrm{~h}$ after injection. All camera heads were equipped with low-energy, high-resolution collimators, and all acquisitions were performed with a $20 \%$ energy window centered at the $159-\mathrm{keV}$ photopeak of ${ }^{123} \mathrm{I}$.

The heart-to-mediastinum $(\mathrm{H} / \mathrm{M})$ ratio was determined from the mean counts per pixel in a visually drawn region of interest over the cardiac silhouette divided by the mean counts per pixel in a $7 \times 7$ pixel region of interest placed in the upper mediastinum, using ImageJ software, version 1.49 (National Institutes of Health). The H/M ratio was determined using both image sets for each study; H/M ratios from the 4-h images are reported in the following text. The washout rates were determined from the change in $\mathrm{H} / \mathrm{M}$ ratio from the 15-min to the 4-h planar images using the standard formula: [(early heart counts/ pixel - early mediastinum counts/pixel) - (late heart counts/pixel late mediastinum counts/pixel)]/(early heart counts/pixel - early mediastinum counts/pixel) $\times 100(21)$. The process was repeated for both pre- and postablation ${ }^{123} \mathrm{I}$-MIBG studies.

The 4-h reconstructed short-axis images were integrated in PMOD software, version 3.4 (PMOD Technologies), which allowed semiquantitative analysis of the ${ }^{123}$ I-MIBG images. A standard 17-segment model and a novel 757-segment model were generated for every study. The 757segment model is a semiquantitative ${ }^{123} \mathrm{I}-\mathrm{MIBG}$ analysis tool resulting in a polar plot of the left ventricle that is formed of 21 circular slices plus the apex divided into 36 rows separated by $10^{\circ}$-angle increments. Both the preablation and postablation 757-segment models were normalized to the highest uptake segment in their respective 17-segment model.

To evaluate changes after VT ablation, the pre- and postablation 757segment polar plots were uploaded into CardioView (QRS Diagnostic), a custom cardiac software viewing and analysis tool that directly overlays the electrophysiologic voltage points and ablation sites onto the corresponding ${ }^{123} \mathrm{I}-\mathrm{MIBG}$ segments. The electrophysiologic map was coregistered with the ${ }^{123}$ I-MIBG polar plot after determining the $0^{\circ}, 90^{\circ}, 180^{\circ}, 270^{\circ}$, and apical voltage control points in the electrophysiologic map. For specific VT ablation sites targeted with activation or pace mapping, ${ }^{123}$ I-MIBG uptake was determined in the pre- and postablation studies, enabling assessment of the regional ${ }^{123} \mathrm{I}-\mathrm{MIBG}$ uptake changes after the procedure.

\section{Innervation Zone Definitions and Determination}

${ }^{123}$ I-MIBG denervated (DZ), transitional (TZ), and normally innervated (NZ) zones were first visually determined by 2 independent experienced nuclear medicine physicians (Fig. 1), with disagreement resolved by consensus. This cohort served as a derivation cohort to quantify the visually determined areas of DZ, TZ, and NZ by measuring the mean imaging intensity for each of the innervation categories and to determine the best cutoffs using receiver operating characteristic curves. To assess intra- and interpersonal variability of the visual delineation of the three ${ }^{123} \mathrm{I}-\mathrm{MIBG}$ zones, the TZ was delineated visually by 2 independent operators on 2 occasions each in 10 randomly selected patients. The mean ${ }^{123} \mathrm{I}-\mathrm{MIBG}$ uptake in each of the 757 segments was calculated both before and after ablation, as was the difference between the 2 studies. Each of the segments was then defined as being DZ, TZ, or NZ.

\section{Statistics}

SPSS, version 22.0 (IBM), for Windows (Microsoft) was used for statistical analyses. Continuous variables are expressed as median and quartiles (Q1-Q3) unless otherwise noted. Comparisons were conducted using a 2-tailed nonparametric Mann-Whitney $U$ test for nonpaired and the Wilcoxon signed rank test for paired comparisons. Differences were considered significant at a $P$ value of less than 0.05 .

\section{RESULTS}

\section{Patient Characteristics}

Thirteen patients underwent ${ }^{123}$ I-MIBG scans before radiofrequency ablation of drug-refractory VT (Table 1). The preprocedural ${ }^{123} \mathrm{I}-\mathrm{MIBG}$ scans were performed $5 \mathrm{~d}(\mathrm{Q} 1-\mathrm{Q} 3,2-9 \mathrm{~d})$ before the VT ablation and were repeated after $190 \mathrm{~d}$ (Q1-Q3, 181-203 d). Nine of $13(69 \%)$ patients had ICM. The average patient age at time of enrollment was 62 y (Q1-Q3, 56-73 y), and 100\% were male. 


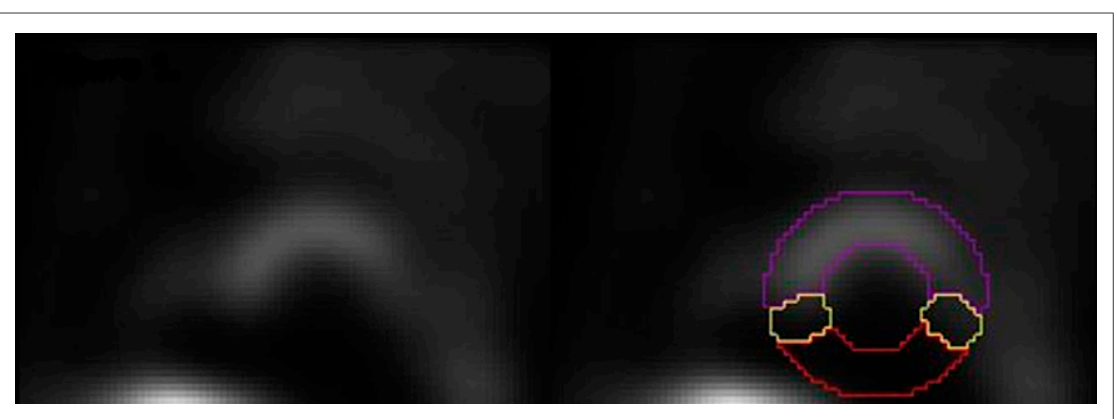

FIGURE 1. Cardiac ${ }^{123}$ I-MIBG short-axis SPECT image shows inferior denervation with visua outline of DZ (red), TZ (yellow), and NZ (purple). These assessments were performed by 2 experienced operators, with disagreements resolved via discussion.

Median left ventricular ejection fraction was 30\% (Q1-Q3, 20\%$35 \%)$. All patients had an ICD in place.

\section{I-MIBG Analysis}

For planar images, 2 operators each independently measured $\mathrm{H} / \mathrm{M}$ twice on a total of 4 separate days, resulting in less than $10 \%$ intra- and interpersonal variations. Identical regions of interest were used in 8 patients $(62 \%)$ and were modified to best fit in the remaining 5 patients. $\mathrm{H} / \mathrm{M}$ ratio and washout rate for all patients were

TABLE 1

Characteristics of 13 Male Patients Who Underwent ${ }^{123}$ I-MIBG Scans Before Radiofrequency Ablation of Drug-Refractory VT

\begin{tabular}{lc}
\hline \multicolumn{1}{|c}{ Patient characteristic } & Value \\
\hline Cardiomyopathy $(n)$ & $9(69 \%)$ \\
\hline ICM & $4(31 \%)$ \\
\hline NICM & 62 \\
\hline Age at time of ablation $(y)$ & \\
Ejection fraction (\%) & 30 \\
Median & $56-73$ \\
Q1-Q3 & $13(100 \%)$ \\
\hline Presence of ICD at time & \\
of ablation $(n)$ & $3(23.1 \%)$ \\
\hline Prior ablations $(n)$ & \\
\hline Comorbidities $(n)$ & $5(38.4 \%)$ \\
\hline Diabetes & $10(76.9 \%)$ \\
\hline Hypertension & $7(53.8 \%)$ \\
\hline Hyperlipidemia & $3(23.0 \%)$ \\
\hline Atrial fibrillation & \\
\hline Medications $(n)$ & $11(84.6 \%)$ \\
\hline ACEI/ARB & $11(84.6 \%)$ \\
\hline$\beta$ blocker & $3(23.0 \%)$ \\
\hline Aldosterone antagonist & $11(84.6 \%)$ \\
\hline Amiodarone & $3(23.0 \%)$ \\
\hline Other antiarrhythmic drugs &
\end{tabular}

ACEI = angiotensin-converting-enzyme inhibitor; $\mathrm{ARB}=$ angiotensin receptor blocker. preserved at baseline (1.8 [Q1-Q3, 1.7-2.4] and 54\% [Q1-Q3, 47\%-67\%]) and did not change significantly at the 6-mo follow-up (1.9 [Q1-Q3, 1.6-2.2], $P=0.9$; and 56\% [Q1-Q3, 41\%-62\%], $P=0.6$ ). The H/M ratio before ablation for patients with ICM was 1.7 (Q1-Q3, 1.6-1.8) compared with 1.9 (Q1-Q3, 1.5-2) after ablation $(P=$ $0.57)$. The $\mathrm{H} / \mathrm{M}$ ratio before ablation for patients with NICM was 2.5 (Q1-Q3, 2.32.7) compared with 2.3 (Q1-Q3, 2-2.5) after ablation $(P=0.60)$. Washout rate in patients with ICM was $62 \%$ (Q1-Q3, $54 \%-68 \%)$ before ablation compared with $59 \%$ (Q1-Q3, 41\%-71\%) after ablation $(P$ $=0.35)$. In NICM patients, the washout rate before ablation was $45 \%(\mathrm{Q} 1-\mathrm{Q} 3,42 \%-48 \%)$, and after ablation it was $51 \%(\mathrm{Q} 1-\mathrm{Q} 3,43 \%-56 \%)(P=0.74)$.

Innervation zone determinations were based on the visually determined innervation categories on short-axis SPECT images. In 10 randomly selected patients, this visual determination was performed twice by 2 separate operators, resulting in less than $10 \%$ intra- and interpersonal variation. From these assessments, normalized ${ }^{123}$ I-MIBG uptake in areas of the DZ was $25 \%$ (Q1Q3, 15.3\%-31.7\% [minimum-maximum, 4\%-50\%]) and increased to $40 \%$ (Q1-Q3, 30.2\%-43.6\% [14\%-72\%]) in the TZ $(P<0.001)$. Tracer uptake was highest in myocardium with preserved sympathetic innervation: 67\% (Q1-Q3, 52.2\%-71.4\% [39\%-100\%] $)(P<0.001)$. Receiver operating characteristic curves (Fig. 2) showed that the best cutoff was $30.5 \%$ to distinguish DZ/TZ (sensitivity, 71\%; specificity, 69\%; area under the curve, $0.81 ; 95 \%$ confidence interval, $0.72-0.89)$ and $47.1 \%$ to distinguish TZ/NZ (sensitivity, 89\%; specificity, 82\%; area under the curve, 0.92 ; $95 \%$ confidence interval, 0.85-0.98). These cutoffs were used in the following quantitative segmental analysis (DZ $<30.5 \%$, TZ 30.6\%-47.1\%, and NZ $>47.1 \%$ ).

17-Segment Analysis. When considering the traditional 17segment model, the median segmental ${ }^{123}$ I-MIBG uptake for all patients was $52 \%$ before VT ablation (Q1-Q3, 50\%-62\%) versus $48 \%$ after VT ablation $(\mathrm{Q} 1-\mathrm{Q} 3,46 \%-56 \%)(P=0.42)$. For ICM patients alone, it was $50 \%(\mathrm{Q} 1-\mathrm{Q} 3,48 \%-53 \%)$ versus $47 \%$ (Q1-Q3, 43\%-48\%) $(P=0.06)$. Patients with NICM had ${ }^{123} \mathrm{I}-\mathrm{MIBG}$ uptake of 68\% before VT ablation (Q1-Q3, 63\%-70\%) and 61\% after ablation $(\mathrm{Q} 1-\mathrm{Q} 3,56 \%-68 \%)(P=0.78)$.

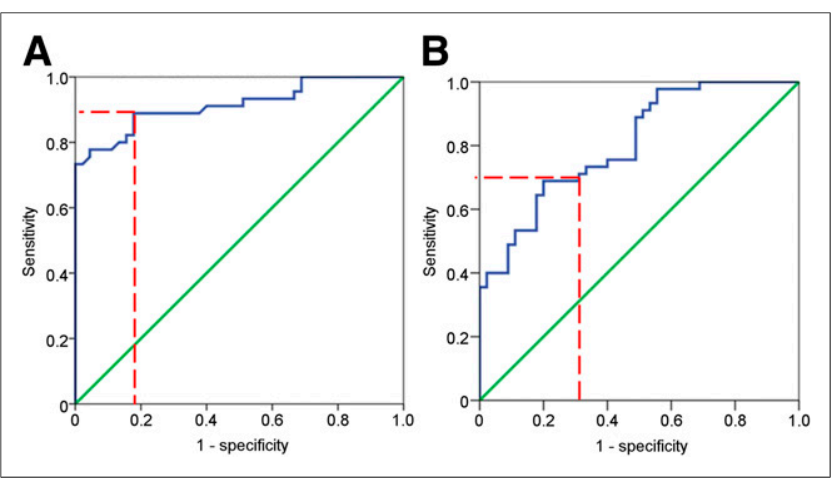

FIGURE 2. A receiver operating characteristic curve shows best cutoff (dashed red line) of ${ }^{123}$ I-MIBG in NZ vs. TZ (A) and DZ vs. TZ (B). 


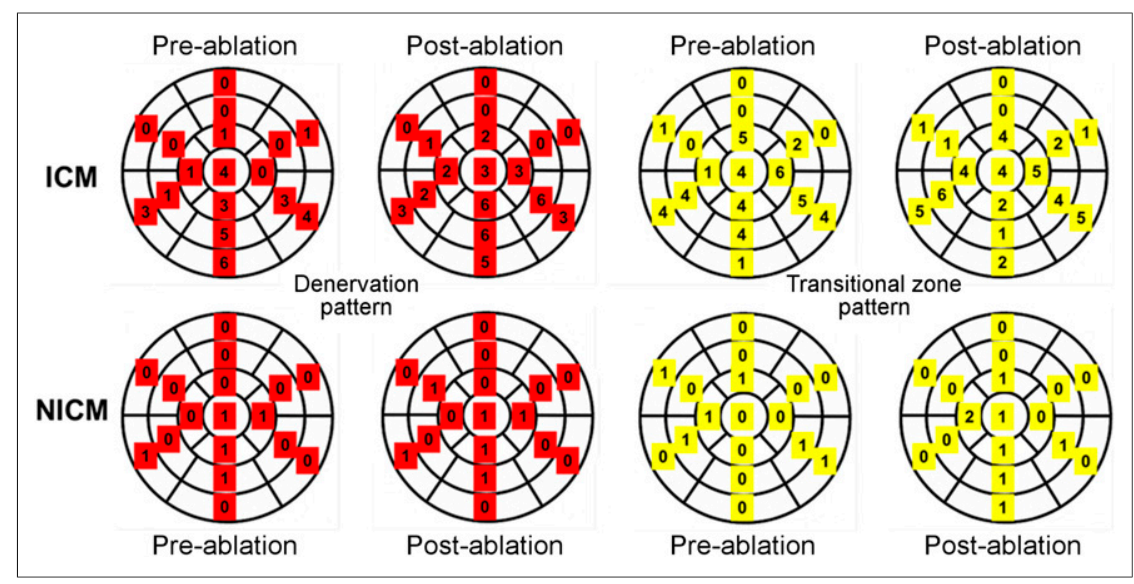

FIGURE 3. Seventeen-segment DZ (red) and TZ (yellow) patterns before and after ablation with numbers of patients affected in each segment.

The percentage of DZ segments (uptake $<30.6 \%$ ) for all patients before and after VT ablation was $18 \%(\mathrm{Q} 1-\mathrm{Q} 3,5.9 \%-$ $29.4 \%)$ and $29 \%(\mathrm{Q} 1-\mathrm{Q} 3,5.9-35.2)(P=0.28)$, respectively. Considering patients with ICM only, it was $22 \%$ (Q1-Q3, 12\%$35 \%)$ and $35 \%(\mathrm{Q} 1-\mathrm{Q} 3,29 \%-41 \%)(P=0.26)$, respectively, whereas in NICM patients, it was 3\% (Q1-Q3, 0\%-8\%) and 6\% (Q1-Q3, 0\%-16\%) $(P=0.61)$, respectively.

In ICM patients, the preablation denervation regions were inferior $(100 \%)$, apical (78\%), lateral (67\%), septal (44\%), and anterior $(11 \%)$; and postablation regions were inferior $(100 \%)$, apical $(78 \%)$, lateral (67\%), septal (67\%), and anterior (33\%) (further details are shown in Fig. 3). For NICM patients, the preablation denervation regions were inferior (75\%), apical (50\%),

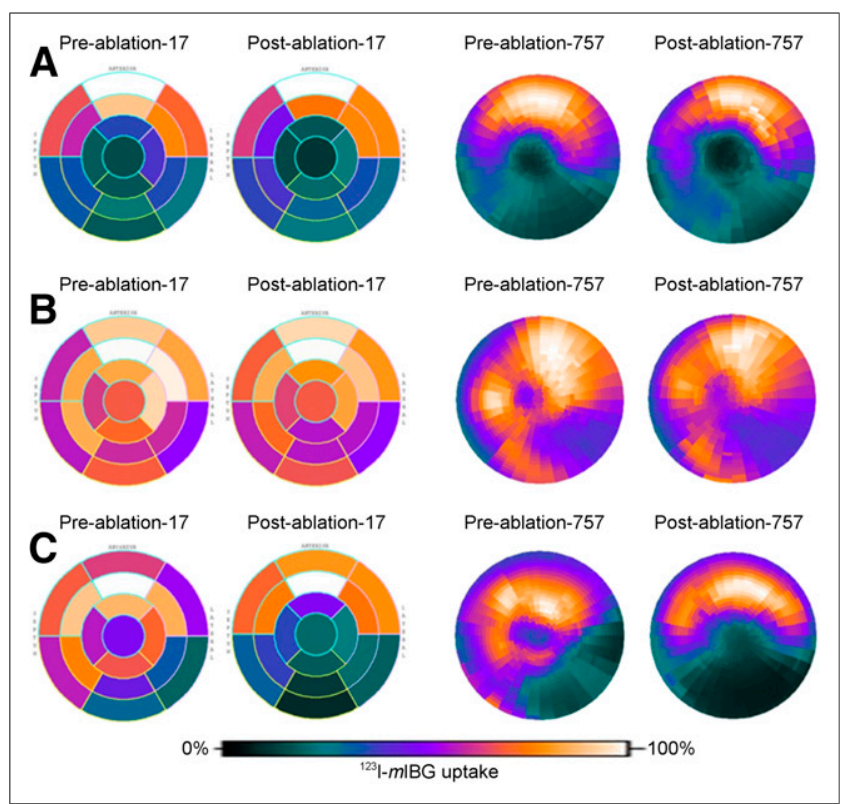

FIGURE 4. Polar plots of standard 17-segment and 757-segment 123I-MIBG uptake at baseline and after VT ablation. (A) Plots of ICM patient at baseline with no significant changes after VT ablation. (B) Plots of NICM patient at baseline with no significant changes after VT ablation. (C) Plots of ICM patient at baseline and after VT ablation showing increasing areas of low MIBG uptake (denervation). lateral $(50 \%)$, septal $(50 \%)$, and anterior $(0 \%)$; and postablation regions were inferior $(75 \%)$, apical $(25 \%)$, lateral $(25 \%)$, septal $(50 \%)$, and anterior $(50 \%)$. After VT ablation, denervation was mostly seen in the area of preablation denervation and in adjacent segments. Inferior innervation defects were seen in all patients with ischemic cardiomyopathy, affecting $40 \%$ of the 63 inferior segments; but such defects were seen in only $75 \%$ of nonischemic patients, affecting $10 \%$ of the 28 inferior segments.

757-Segment Analysis. The 757-segment analysis was successfully performed in all 13 patients (Fig. 4). Of the 9,841 segments analyzed for all patients before undergoing VT ablation, DZ, TZ, and NZ segments accounted for $21 \%(n=2,086), 21 \%(n=$ $2,059)$, and $58 \%(n=5,696)$, respectively. After ablation, these values changed nonsignificantly to $27 \%(n=$ 2,686), 20\% ( $n=2016)$, and 52\% $(n=5,139)$, respectively. ICM patients had a significantly higher percentage of abnormally innervated myocardium $(\mathrm{DZ}+\mathrm{TZ})$ than did NICM patients both before $(52.1 \%$ vs. $19.6 \%, P=0.011)$ and after $(56.7 \%$ vs. $27.5 \%, P=$ 0.011) ablation (Fig. 5).

Dynamic changes from before to after ablation were seen at the segmental level in all patients. There were changes in the designated innervation categories from baseline to the 6-mo follow-up for ischemic (19\% DZ, 59\% TZ, 22\% NZ) and nonischemic (6\% DZ, $45 \% \mathrm{TZ}, 15 \% \mathrm{NZ}$ ) patients (Fig. 6). The proportional highest changes occurred in the TZ. In ischemic patients, relative changes were significantly greater in the TZ segments than in the DZ segments, which had the second highest proportional changes $(P=0.028)$.

\section{VT Ablation and Clinical Follow-up}

A total of 48 different VTs were induced during the ablation procedures for the 13 patients; $60 \%$ were left bundle branch block morphology and $40 \%$ were right bundle branch block morphology. The median cycle length of the VTs was $334 \mathrm{~ms}$ (Q1-Q3, 286$451 \mathrm{~ms})$. Of the $48 \mathrm{VT}$ morphologies induced, 8 clinical VTs were targeted, and 7 (88\%) VTs were rendered noninducible after ablation. Of the 13 patients, 9 patients' ablations were performed in the inferior (7), septal (1), and apical (1) left ventricular wall. Four patients had an ablation performed in a region of the right ventricle: septal (2), inferior (1), and apical (1). Eight patients (62\%) had an exact ablation site with good pace map match for the clinical VT. ${ }^{123}$ I-MIBG uptake for segments correlating to the successful ablation sites was $37.5 \%$ in the preablation studies (Q1-Q3, 22.3\%-59.8\%) and 39.2\% in the postablation studies (Q1-Q3, 18.2\%-64.2\%) $(P=0.99)$. Four patients $(75 \%$ of them with ICM) had their ablation done in the ${ }^{123}$ I-MIBG denervated zone, whereas the other 4 (75\% NICM) had the ablation done in the normally innervated myocardium. The remaining 5 patients had only substrate modification, which did not allow defining a specific segment. Of the 993 total ablation lesions applied to the myocardium in all patients, $788(79 \%)$ were in the DZ, 54 (5\%) were in the TZ, and $151(15 \%)$ were in the NZ.

Of the 10 patients who underwent device interrogation 6 mo after ablation, 3 (30\%) had recurrent VT (including nonsustained VT), and all received appropriate ICD therapies. The remaining 3 patients did not report any symptoms of VT or ICD shocks. VT 


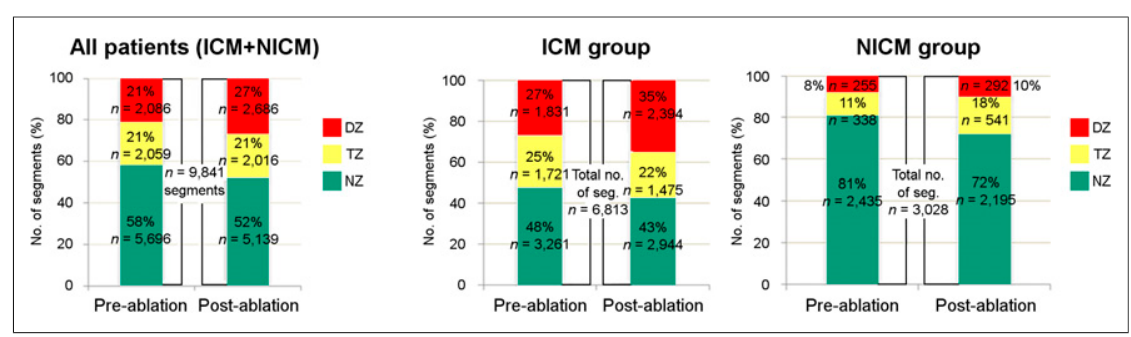

FIGURE 5. 757-segment analysis of three ${ }^{123}$ I-MIBG categories, NZ, TZ, and DZ segments, at baseline and after VT ablation.

recurrence occurred $54 \mathrm{~d}(\mathrm{Q} 1-\mathrm{Q} 3,47-99 \mathrm{~d})$ after ablation. Of the 3 patients with recurrence, one had been noninducible at the conclusion of the procedure; the other 2 had extensive substrate modification to the area thought to be the origin of the VT. The one patient had his VT recurrence later (exactly $143 \mathrm{~d}$ after ablation) than the other 2 patients (54 and $40 \mathrm{~d}$ after ablation). All recurrences occurred in patients with ICM. The mean number of ICD therapies was 2 (Q1-Q3, 1.5-2.5); all were antitachycardia pacing with no ICD shocks. Patients with VT recurrence demonstrated a DZ and TZ of $45 \%$ and $33 \%$ versus $22 \%(P=0.3)$ and $16 \%$ $(P=0.2)$ in patients without recurrence.

\section{DISCUSSION}

Our study has several important findings. An arrhythmiaspecific 757-segment analysis can be used for ${ }^{123}$ I-MIBG analysis, and these segments can be defined quantitatively to distinguish between NZ, DZ, and TZ. Patients with ICM had a larger area of abnormal innervation than patients with NICM. Additionally, VT ablation did not result in a change in global innervation; however, dynamic changes in innervation categories were seen on the segmental innervation level.

A strong relationship exists between sympathetic innervation abnormalities and VT (10-12). Multiple possible mechanisms

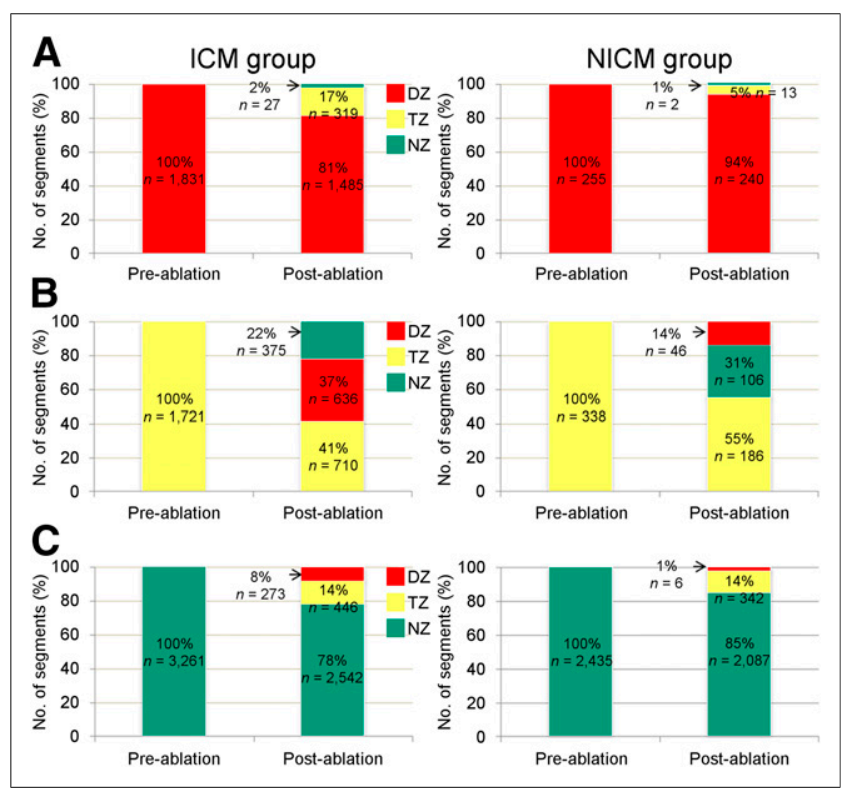

FIGURE 6. Segmental changes in ICM and NICM. Graphs show segments at baseline and changes that occurred for same segments after VT ablation for DZ (A), TZ (B), and NZ (C). have been postulated. Decreased reuptake by impaired myocardial presynaptic nerve terminals results in the buildup of catecholamines in the synaptic cleft, with downregulation of postsynaptic $\beta$-adrenergic receptors being thought to increase arrhythmogenesis $(15,22-25)$. Additionally, denervated but viable myocardium may demonstrate an exaggerated response to circulating catecholamines (10), which may further increase proarrhythmogenicity $(15,19,24,26,27)$. The fact that $\beta$ blockers can protect against the development of VT supports this theory (28). Further evidence of the relationship comes from another recent study suggesting that left and bilateral stellate ganglion blocks decrease ICD shocks (29).

Cardiac sympathetic innervation can be visualized using ${ }^{123} \mathrm{I}-$ MIBG, a norepinephrine analog radiotracer that undergoes reuptake and storage in the presynaptic nerve terminal and is released into the synaptic cleft on the basis of the level of sympathetic input (30). Reduced ${ }^{123}$ I-MIBG uptake has been demonstrated in patients with various cardiomyopathies who experienced VT, and even in normal heart VT (30-33). Global cardiac sympathetic innervation can be assessed using the $\mathrm{H} / \mathrm{M}$ ratio and the washout rate. Multiple studies using these global parameters to evaluate cardiac sympathetic innervation have demonstrated that innervation is associated with ICD therapies, ventricular arrhythmias, and sudden cardiac death $(16,30)$.

There are several shortcomings with current assessments of cardiac sympathetic innervation using global parameters and the 17-segment model. Loss of sympathetic innervation is regional and heterogeneous, which limits the usefulness of global parameters. The current 17 -segment model was designed with coronary artery distribution in mind, in which localization of perfusion to portions of the 3 major coronary artery distributions is needed (18). In the current study, we applied a novel, arrhythmia-specific, 757-segment model of cardiac sympathetic innervation, with low inter- and intraobserver variability attesting to the method's reproducibility. Analysis at this resolution will provide important pathophysiologic insights.

Prior quantitative definitions of levels of innervation as assessed by ${ }^{123}$ I-MIBG SPECT scans did not exist. Without standard definitions, comparisons in prospective clinical and research studies would remain challenging. To that end, we defined cutoff points based on normalized values assigned to each segment. Using receiver operating characteristic curves as described previously, reasonable sensitivities and specificities were obtained for values defining the DZ-TZ boundary as well as the TZ-normal innervation boundary.

In addition, important differences were found between patients with ICM and those with NICM. ICM patients had more segments with abnormal innervation at baseline and the 6-mo follow-up than did NICM patients. This is despite there being no statistically significant differences between the severity of left ventricular dysfunction as ascertained by the left ventricular ejection fraction (30\% vs. $25 \%, P=$ not significant). Although multiple trials assessing the prognostic value of ${ }^{123}$ I-MIBG have looked at patients with both NICM and ICM (20), and some studies have focused exclusively on patients without coronary artery disease $(33,34)$, still little is known about the differences in sympathetic innervation in these disease states. One important difference found in this study is that large inferior innervation defects were seen in 
all patients with ischemic cardiomyopathy but that some patients with nonischemic disease were spared and defects, if present, affected less of the inferior wall, suggesting that they were not artifacts. Also of interest, all 4 of the mapped ablation sites in normal myocardium were in patients with NICM, suggesting that the interaction between cardiac sympathetic innervation and VT may differ among the different types of cardiomyopathies. Future imaging studies in a larger number of subjects are needed to better understand the differences in sympathetic denervation between patients with ICM and those with NICM.

Global cardiac sympathetic innervation did not significantly change after VT ablation. Nor were significant local innervation changes noted at the specific ablation sites, which may be because VT ablation was mainly performed in the already-denervated segments of the myocardium. However, dynamic changes were seen regionally in the postablation studies distant from ablation sites, supporting the importance of a high-resolution segmental analytic approach. Changes distant from points of ablation may have a variety of causes, including downstream effects of ablation on proximal nerve sites, changes in the ventricular milieu due to decreased arrhythmia burden, or other unknown feedback mechanisms that contribute to the regulation of cardiac sympathetic innervation.

Regional changes affected all 3 innervation categories but were proportionally highest in the TZ, possibly indicating an area with increased innervation instability. Changes even occurred in the DZ, affecting up to $19 \%$ and $6 \%$ of segments in ICM and NICM, respectively. These findings may reflect a difference between truly damaged sympathetic innervation versus the possibility of "stunned" innervation, similar in concept to hibernating myocardium that can regain function after successful revascularization $(34,35)$. This research offers new pathophysiologic insights into the process of denervation and its impact on clinical outcomes, including VT and sudden cardiac death.

To our knowledge, ours is the first feasibility study exploring the effect of VT radiofrequency ablation on global and regional cardiac sympathetic innervation and its possible link to the recurrence of VT. Although statistically not significant because of the small number of subjects studied, our findings are interesting in that patients with VT recurrence had a nearly twofold larger area of abnormal innervation than patients without VT recurrence. This finding is consistent with prior studies assessing the effects of cardiac sympathetic innervation on the risk of VT (32). Our findings may indicate that a larger TZ could predict VT recurrence after ablation, which will need to be confirmed in larger studies. Future studies with a larger sample size will hopefully be able to better correlate imaging features such as total denervation or dynamic innervation changes with long-term clinical outcome.

Our study has several important limitations. This is a singlecenter study with a small sample size; larger studies are required to better understand the differences in sympathetic denervation between ICM and NICM. Women are underrepresented in this trial, and it is possible that the cutoff points for the different innervation zones may differ between men and women. A control group to compare the longitudinal changes of cardiac innervation in a similar cardiac patient population not undergoing VT ablation was not available for this feasibility study but needs to be part of future trials. Medication changes, temporal changes, and other interventions that may affect cardiac innervation were not controlled for. Because of the low ${ }^{123}$ I-MIBG tracer count, the exact delineation of myocardium can be challenging in some patients and was resolved only after careful, consensus review by the 2 expert reviewers. Innervation heterogeneity has been described in apparently healthy subjects, raising the possibility of regionally variable thresholds $(36,37)$. However, described variations were above the denervation threshold defined in this study. Although great effort was taken to ensure similar alignment of the pre- and postimaging datasets and registration of the electrophysiology datasets, it is impossible to exclude that registration errors could affect the global or regional analysis.

\section{CONCLUSION}

Patients with ischemic cardiomyopathy have significantly larger areas of abnormal innervation than those with nonischemic cardiomyopathy. Although VT ablation did not change global innervation, a novel arrhythmia-specific segmental analysis demonstrated significant dynamic changes in innervation categories and allowed quantitative definitions of DZ, TZ, and NZ. These findings provide new insights into the mechanics of sympathetic innervation in patients undergoing VT ablation and may have diagnostic and therapeutic implications.

\section{DISCLOSURE}

This work was supported by research grants from GE Healthcare and Biosense Webster. No other potential conflict of interest relevant to this article was reported.

\section{REFERENCES}

1. Zipes DP, Camm AJ, Borggrefe M, et al. ACC/AHA/ESC 2006 guidelines for management of patients with ventricular arrhythmias and the prevention of sudden cardiac death: a report of the American College of Cardiology/American Heart Association Task Force and the European Society of Cardiology Committee for Practice Guidelines (writing committee to develop guidelines for management of patients with ventricular arrhythmias and the prevention of sudden cardiac death): developed in collaboration with the European Heart Rhythm Association and the Heart Rhythm Society. Circulation. 2006;114:e385-e484.

2. deBakker JM, van Capelle FJ, Janse MJ, et al. Macroreentry in the infarcted human heart: the mechanism of ventricular tachycardias with a "focal" activation pattern. J Am Coll Cardiol. 1991;18:1005-1014

3. Stevenson WG, Wilber DJ, Natale A, et al. Irrigated radiofrequency catheter ablation guided by electroanatomic mapping for recurrent ventricular tachycardia after myocardial infarction: the multicenter thermocool ventricular tachycardia ablation trial. Circulation. 2008;118:2773-2782.

4. Dickfeld T, Lei P, Dilsizian V, et al. Integration of three-dimensional scar maps for ventricular tachycardia ablation with positron emission tomography-computed tomography. JACC Cardiovasc Imaging. 2008;1:73-82.

5. Dickfeld T, Tian J, Ahmad G, et al. MRI-guided ventricular tachycardia ablation: integration of late gadolinium-enhanced 3D scar in patients with implantable cardioverter-defibrillators. Circ Arrhythm Electrophysiol. 2011;4:172-184.

6. Klein T, Abdulghani M, Smith M, et al. Three-dimensional ${ }^{123}$ I-meta-iodobenzylguanidine cardiac innervation maps to assess substrate and successful ablation sites for ventricular tachycardia: a feasibility study for a novel paradigm of innervation imaging. Circ Arrhythm Electrophysiol. February 23, 2015 [Epub ahead of print].

7. Tian J, Jeudy J, Smith MF, et al. Three-dimensional contrast-enhanced multidetector CT for anatomic, dynamic, and perfusion characterization of abnormal myocardium to guide ventricular tachycardia ablations. Circ Arrhythm Electrophysiol. 2010;3:496-504.

8. Tian J, Smith MF, Ahmad G, Dilsizian V, Jimenez A, Dickfeld T. Integration of 3-dimensional scar models from SPECT to guide ventricular tachycardia ablation. J Nucl Med. 2012;53:894-901.

9. Tian J, Smith MF, Chinnadurai P, et al. Clinical application of PET/CT fusion imaging for three-dimensional myocardial scar and left ventricular anatomy during ventricular tachycardia ablation. J Cardiovasc Electrophysiol. 2008; 20:597-604.

10. Podrid PJ, Fuchs T, Candinas R. Role of the sympathetic nervous system in the genesis of ventricular arrhythmia. Circulation. 1990;82:1103-1113. 
11. Weiss JN, Nademanee K, Stevenson WG, Singh B. Ventricular arrhythmias in ischemic heart disease. Ann Intern Med. 1991;114:784-797.

12. Zipes DP. Sympathetic stimulation and arrhythmias. N Engl J Med. 1991;325: 656-657.

13. Chen W, Cao Q, Dilsizian V. Variation of heart-to-mediastinal ratio in ${ }^{123} \mathrm{I}-\mathrm{mIBG}$ cardiac sympathetic imaging: its affecting factors and potential corrections. Curr Cardiol Rep. 2011;13:132-137.

14. Dilsizian V, Chandrashekhar Y, Narula J. Introduction of new tests: low are the mountains, high the expectations. JACC Cardiovasc Imaging. 2010;3:117-119.

15. Verrier RL, Antzelevitch C. Autonomic aspects of arrhythmogenesis: the enduring and the new. Curr Opin Cardiol. 2004;19:2-11.

16. Zhou W, Chen J. I-123 metaiodobenzylguanidine imaging for predicting ventricular arryhthmia in heart failure patients. J Biomed Res. 2013;27: 460-466.

17. Al Badarin FJ, Wimmer AP, Kennedy KF, Jacobson AF, Bateman TM. The utility of ADMIRE-HF risk score in predicting serious arrhythmic events in heart failure patients: incremental prognostic benefit of cardiac ${ }^{123} \mathrm{I}-\mathrm{mIBG}$ scintigraphy. J Nucl Cardiol. 2014;21:756-762.

18. Cerqueira MD, Weissman NJ, Dilsizian V, et al. Standardized myocardial segmentation and nomenclature for tomographic imaging of the heart: a statement for healthcare professionals from the Cardiac Imaging Committee of the Council on Clinical Cardiology of the American Heart Association. Circulation. 2002;105: 539-542.

19. Dae MW, O'Connell JW, Botvinick EH, Chin MC. Acute and chronic effects of transient myocardial ischemia on sympathetic nerve activity, density, and norepinephrine content. Cardiovasc Res. 1995;30:270-280.

20. Jacobson AF, Senior R, Cerqueira MD, et al. Myocardial iodine-123 metaiodobenzylguanidine imaging and cardiac events in heart failure: results of the prospective ADMIRE-HF (AdreView Myocardial Imaging for Risk Evaluation in Heart Failure) study. J Am Coll Cardiol. 2010;55:2212-2221.

21. Ogita H, Shimonagata T, Fukunami M. Prognostic significance of cardiac ${ }^{123}$ I-metaiodobenzylguanidine imaging for mortality and morbidity in patients with chronic heart failure: a prospective study. Heart. 2001;86: $656-660$.

22. Spyrou N, Rosen SD, Fath-Ordoubadi F, et al. Myocardial beta-adrenoreceptor density one month after acute myocardial infarction predicts left ventricular volumes at six months. J Am Coll Cardiol. 2002;40:1216-1224.

23. Choudhry L, Rosen SD, Lefroy DC, Nihoyannopoulos P, Oakley CM, Camici PG. Myocardial beta adrenoceptor density in primary and secondary left ventricular hypertrophy. Eur Heart J. 1996;17:1703-1709.
24. Schäfers M, Wichter T, Lerch H, et al. Cardiac ${ }^{123} \mathrm{I}-\mathrm{MIBG}$ uptake in idiopathic ventricular tachycardia and fibrillation. J Nucl Med. 1999;40:1-5.

25. Caldwell JH, Link JM, Levy WC, Poole JE, Stratton JR. Evidence for pre- to postsynaptic mismatch of the cardiac sympathetic nervous system in ischemic congestive heart failure. J Nucl Med. 2008;49:234-241.

26. Liu Q, Chen D, Wang Y, Zhao X, Zheng Y. Cardiac autonomic nerve distribution and arrhythmia. Neural Regen Res. 2012;7:2834-2841.

27. Schäfers J, Lerch H, Wichter T, et al. Cardiac sympathetic innervation in patients with idiopathic right ventricular outflow tract tachycardia. J Am Coll Cardiol. 1998;32:181-186.

28. Packer M, Bristow MR, Cohn JN, et al. The effect of carvedilol on morbidity and mortality in patients with chronic heart failure: U.S. Carvedilol Heart Failure Study Group. N Engl J Med. 1996;334:1349-1355.

29. Vaseghi M, Gima J, Kanaan C, et al. Cardiac sympathetic denervation in patients with refractory ventricular arrhythmias or electrical storm: intermediate and long-term follow-up. Heart Rhythm. 2014;11:360-366.

30. Klein T, Dilsizian V, Cao Q, Chen W, Dickfeld TM. The potential role of iodine123 metaiodobenzylguanidine imaging for identifying sustained ventricular tachycardia in patients with cardiomyopathy. Curr Cardiol Rep. 2013;15:359.

31. Li ST, Tack CJ, Fananapazir L, Goldstein DS. Myocardial perfusion and sympathetic innervation in patients with hypertrophic cardiomyopathy. J Am Coll Cardiol. 2000;35:1867-1873.

32. Boogers MJ, Borleffs CJ, Henneman MM, et al. Cardiac sympathetic denervation assessed with 123-iodine metaiodobenzylguanidine imaging predicts ventricular arrhythmias in implantable cardioverter-defibrillator patients. $J$ Am Coll Cardiol. 2010;55:2769-2777.

33. Mitrani RD, Klein LS, Miles WM, et al. Regional cardiac sympathetic denervation in patients with ventricular tachycardia in the absence of coronary artery disease. J Am Coll Cardiol. 1993;22:1344-1353.

34. Verberne HJ, Brewster LM, Somsen GA, van Eck-Smit BL. Prognostic value of myocardial ${ }^{123}$ I-metaiodobenzylguanidine (MIBG) parameters in patients with heart failure: a systematic review. Eur Heart J. 2008;29:1147-1159.

35. Fallavollita JA, Canty JM Jr. Dysinnervated but viable myocardium in ischemic heart disease. J Nucl Cardiol. 2010;17:1107-1115.

36. Morozumi T, Kusuoka H, Fukuchi K, et al. Myocardial iodine-123-metaiodobenzylguanidine images and autonomic nerve activity in normal subjects. $\mathrm{J} \mathrm{Nucl}$ Med. 1997;38:49-52.

37. Gill JS, Hunter GJ, Gane G, Camm AJ. Heterogeneity of the human myocardial sympathetic innervation: in vivo demonstration by iodine 123-labeled metaiodobenzylguanidine scintigraphy. Am Heart J. 1993;126:390-398. 